\title{
Kernos
}

Revue internationale et pluridisciplinaire de religion grecque antique

$13 \mid 2000$

Varia

\section{Elias TEMPELIS, The School of Ammonius, son of Hermias, on Knowledge of the Divine}

\section{Aikaterini Lefka}

\section{OpenEdition \\ Journals}

\section{Édition électronique}

URL : http://journals.openedition.org/kernos/1344

DOI : 10.4000/kernos. 1344

ISSN : 2034-7871

\section{Éditeur}

Centre international d'étude de la religion grecque antique

Édition imprimée

Date de publication : 1 janvier 2000

ISSN : 0776-3824

\section{Référence électronique}

Aikaterini Lefka, «Elias TEMPELIs, The School of Ammonius, son of Hermias, on Knowledge of the Divine », Kernos [En ligne], 13 | 2000, mis en ligne le 21 avril 2011, consulté le 21 septembre 2020. URL : http://journals.openedition.org/kernos/1344 ; DOI : https://doi.org/10.4000/kernos.1344 
En effet, la liturgie mithriaque paraît avoir été, elle aussi, largement empreinte de pratiques magiques; c'est ce que montrent en effet la Mithrasliturgie du grand papyrus magique de Paris ou les invocations faites à Mithra dans certaines formules magiques de guérison. Pour l'A., la «magie » procède d'une connaissance approfondie que les prêtres iraniens, - magoi -; avaient de la nature; cette connaissance se répandit peu à peu hors de la caste des « mages » pour pénétrer dans divers milieux hellénisés, imprégnés de culture grecque et, entre autres, dans les sphères où l'on vénérait Mithra. Favorable à l'interprétation jadis donnée par Cumont, selon qui le mithriacisme serait né en Asie Mineure et aurait été forgé par les magoi, l'A. précise ainsi la part que prit la culture grecque dans le développement du mithriacisme.

Alliant une connaissance approfondie de l'iconographie et des textes anciens à une parfaite maîtrise de la bibliographie, l'A., en n'hésitant jamais à éprouver des hypothèses anciennes, aboutit à une approche résolument nouvelle du mithriacisme. Cet ouvrage constitue une avancée notable dans la connaissance de la religion mithriaque et, plus largement, dans celle des courants philosophicoreligieux de l'hellénisme. Trois index (noms antiques de personnes et lieux, symboles et principaux thèmes, voces magica) complètent utilement ce travail: on regrette de n'y trouver nulle part d'index des noms d'auteurs modernes, outil dont la présence se justifierait d'autant mieux que, depuis les travaux de Cumont, le mithriacisme a nourri une abondante controverse.

Isabelle Tassignon (Université de Liège)

Elias Tempelis, The Scbool of Ammonius, son of Hermias, on Knowledge of

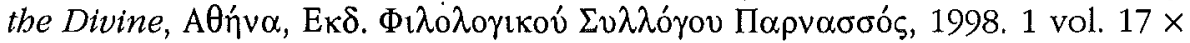
$24 \mathrm{~cm}, 168$ p. ISBN : 960-85212-5-4.

L'ouvrage est une version légèrement modifiée de la thèse de doctorat que l'auteur a soutenue en 1994, à l'Université de Londres. Il s'agit d'une étude sur la méthode et le fondement théorique de la connaissance du divin et du monde intelligible prônés par Ammonius d'Alexandrie et par ses élèves: Asclépius, Jean Philopon, Zacharie, Gessius, Pseudo-Héliodore, l'auteur anonyme du commentaire In de Interpretatione, Olympiodore et ses élèves (Élie, David, Stephanus, Pseudo-Élie, Pseudo-David et l'auteur anonyme du Prolegomena philosophicae "Syllabi » Platonicae).

Du point de vue de l'histoire des religions et de l'histoire de la philosophie, l'école d'Ammonius est intéressante à plus d'un titre. Située à Alexandrie, lieu par excellence d'échanges et de syncrétismes de toutes les traditions philosophiques et religieuses du monde méditerranéen, elle est active entre 485 et 610 de notre ère, à l'époque de la transition définitive du paganisme au christianisme. L'école reste officiellement fidèle à la religion grecque, mais les derniers élèves illustres sont des chrétiens. Sa clôture marque la fin de la longue lignée des écoles philosophiques de l'antiquité grecque. Comment les héritiers de traditions si diversifiées ont-ils affronté les grandes questions métaphysiques? L'idée longuement soutenue, fondée sur des impressions générales, que l'école d'Ammonius n'avait pas développé une doctrine métaphysique propre et qu'elle représentait surtout un effort pour couvrir la distance entre le platonisme et le christianisme a été récemment démentie par K. Verrycken (The Metaphysics of Ammonius, son of Hermeias, in R. Sorabji [ed.], Aristotle Transformed, The Ancient Commentators and their Influence, London-Ithaca-N. York, 1990). M. Tempelis vient pallier l'absence d'une recherche approfondie sur le sujet, tâche déjà déli- 
cate, rendue encore plus difficile par le manque de lexiques spécialisés dans la terminologie philosophique de cette époque.

Le problème méthodologique essentiel, habilement rencontré par l'auteur, est l'absence d'un exposé systématique et complet de la doctrine. Les textes examinés sont des commentaires d'œuvres de philosophes antérieurs: Platon, Aristote, Porphyre, Nicomaque, Hippocrate et Paulus. Comme c'est le cas souvent dans le néoplatonisme, dont fait partie l'école d'Ammonius, un commentateur tend à revenir sur un sujet à propos duquel il s'est forgé un avis personnel, même si le contexte ne s'y prête pas particulièrement. C'est ainsi que le thème de la connaissance du divin revient avec une fréquence impressionnante dans tout le corpus de ces penseurs. Pour reconstruire la théorie de l'école d'Ammonius sur ce sujet, ces informations implicites et disparates ont dû être recueillies et examinées à la lumière de l'ensemble des concepts métaphysiques et épistémologiques exposés (notons que l'étude se limite aux doctrines qui ne sont pas manifestement influencées par le christianisme). L'auteur examine aussi la validité de l'argumentation et les rapports avec la tradition philosophique relative à la question de la connaissance du divin.

En fait, l'école d'Ammonius suit les principes métaphysiques de Proclus et de Plotin, tout en y apportant, comme nous le verrons, des éléments nouveaux et reste liée à l'école d'Athènes par de nombreuses tendances. En commentant des textes des auteurs antérieurs, ces penseurs cherchent surtout à fonder leurs propres concepts. C'est ainsi qu'ils interprètent Aristote afin de prouver qu'il y a cohérence et continuité entre ses doctrines et celles de Platon - lui aussi compris à leur manière. Dans le même esprit, ils exercent une critique dense à l'encontre de l'argumentation métaphysique des philosophes antérieurs, qui «n'avaient pas bien saisi » les enjeux du sujet.

L'auteur articule ses données en trois chapitres : I. Présuppositions et objectif de la connaissance du monde intelligible; II. La connaissance des universaux; III. La connaissance du divin. Le système métaphysique de l'école d'Ammonius accepte la hiérarchie des êtres, en fonction du degré de leur participation aux émanations du divin. Il existe quatre ordres d'entités : a) des substances naturelles; b) des entités psychiques; c) des entités intelligibles; d) du divin.

Au sommet des étants est l'Un, ou l'Hénade, dont la bonté rayonne en créant toute chose. Les entités intelligibles seules sont parfaites, originales, non-composées, éternelles, immobiles; leurs essences sont identiques aux entités elles-mêmes et complètement distinctes de la matière. L'activité qui leur est propre consiste à se retourner vers elles-mêmes. Parmi elles, placées juste en dessous de l'Un, figurent successivement l'Être, la Vie et l'Intellect. L'Être est indépendant de tout et génère l'Intellect, les anges et les objets particuliers. Il est important de noter que

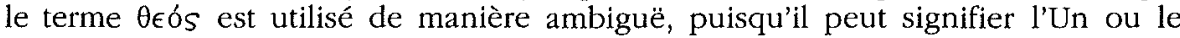
Démiurge, ou les deux à la fois. Le rapport entre le Démiurge, l'Intellect et l'Un n'est pas toujours clair, non plus. Dans l'ordre des intelligibles existent d'autres entités transcendantes : des dieux, avec leur personnalité propre, qui sont également des émanations de l'Un, selon le principe de la différenciation, des anges, des héros, des démons et des nymphes. Les anges sont des activités pures et éternelles, alors que les démons sont exclus de tout, puisqu'ils hantent les bonnes âmes; leur éternité est douteuse.

Concernant les attributs qui caractérisent le divin, l'école d'Ammonius ne présente pas toujours des idées homogènes. Pour la majorité de ces penseurs, le divin est incorporel, ni matériel, ni intelligible, en tant que cause qui transcende la matière et les universaux (entités intelligibles). Il est omniprésent, ni divisible, ni indivisible, il est éternel et en parfaite actualité constante. Il est immuable et 
crée sans cesse par émanations les mondes intelligible et sensible, dont il a l'omniscience. Le Démiurge ne crée pas directement l'ensemble du monde; la hiérarchie ontologique descendant des entités intelligibles aux sensibles et périssables constitue aussi un ordre de causes génératrices successives. Dieu ne peut qu'être la source de tout bien, alors que le mal doit dériver d'ailleurs. Le divin, par respect du libre arbitre de l'homme, peut néanmoins ne pas intervenir pour l'empêcher à faire le mal (l'exposé de ce sujet est trop incomplet dans les textes).

L'âme humaine avait à l'origine une connaissance immédiate du monde intelligible des Formes immuables, vraies et éternelles, dont elle faisait partie. Elle était capable de signifier tout par le moyen de concepts, sans avoir besoin de langage. La situation a changé après l'union de l'âme avec le corps. L'oubli généré par la matérialité rend l'homme ignorant des vérités intelligibles; l'âme garde néanmoins le désir et la potentialité de leur re-connaissance, grâce à sa place intermédiaire entre le sensible et l'intelligible. Tout en restant incapable de connaître directement et pleinement le divin, l'homme essaie d'acquérir autant qu'il lui est possible cette connaissance indirectement, par référence aux créations du divin, en examinant leurs principes et leurs causes. L'Un qui transcende

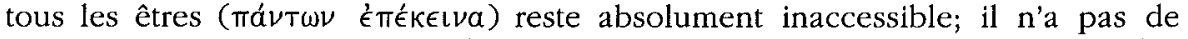
principe et ne participe à rien d'autre que lui-même. Nous ne saurions rien dire sur lui - il n'a pas de nom -, mais nous pouvons parler de son rayonnement, dont dérivent les autres êtres. L'acquisition de la connaissance du monde intelligible ne peut qu'être partielle pour l'homme; le langage, dont il a actuellement besoin, reste inadéquat pour l'exprimer. Toute connaissance possible des entités intelligibles est effectuée par l'activation des principes-raisons cognitives ( $\gamma \nu \omega \sigma \tau l-$

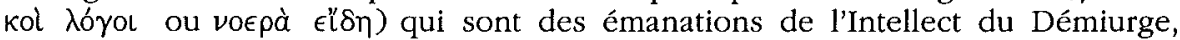
innées à l'âme. Plotin, à qui sont empruntés ces énoncés, s'est contenté de les mentionner. L'école d'Ammonius entreprend d'exposer en détail la procédure de

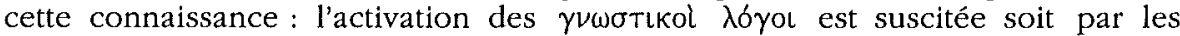
données du monde matériel, perçues par nos sens, qui sont des représentations imparfaites des Formes intelligibles, soit par la parole d'un maître, soit par une illumination. L'âme, elle-même une image de l'Intellect du Démiurge, doit se purger de ses passions, de son imagination ou de ses croyances fausses générées par la perception. Elle se souvient alors des modèles-principes et se rend compte de la différence ontologique entre les mondes sensible et intelligible. À l'aide d'un enseignement et d'une pratique philosophique, l'homme cherche constamment le principe unifiant derrière le multiple, c'est-à-dire le divin.

La connaissance du divin est la première étape d'une procédure qui vise, dans un deuxième temps, à l'assimilation de l'âme à lui. C'est par l'exercice de la raison et par l'intuition, qui n'exigent ni données de sens, ni imagination et sont indépendantes du corps, que l'homme a l'espoir de contempler de son mieux le monde intelligible, et donc le divin. Cette contemplation peut s'accomplir à un moment privilégié ou son âme est séparée du corps (après la mort ou dans cette vie grâce à des événements rarissimes : une illumination ou une possession divine). C'est alors que l'intellect humain atteint sa perfection. Les rares personnes qui ont pu arriver à ce niveau au long d'une vie pure et suite à une pratique prolongée de la philosophie ont atteint l'objectif suprême, l'assimilation au divin. Ils acquièrent alors les attributs du divin : la bonté, l'omniscience et la force. Cet idéal est surtout éthique; quand il se réalise, le philosophe devient un Sage. Il se montre provident pour les âmes des autres, par exemple en participant aux actes politiques de juridiction ou de législation. La participation aux affaires humaines ne constitue pas un besoin pour lui. Il s'agit plutôt d'un acte de bonté, car l'activation complète des raisons-principes de son intellect et l'assimilation au dieu suffisent à son propre bonheur. 
La justice de la providence divine s'exprime également dans le fait que l'âme humaine est immortelle : après la mort, chacun reçoit ainsi le sort qui lui est dû, en fonction de la manière dont il a vécu. La doctrine eschatologique de l'école n'étant pas complète, nous savons seulement que les âmes mauvaises doivent se purifier de leurs fautes, avant de retrouver leur état naturel.

En conclusion, l'auteur remarque que la théorie de l'école d'Ammonius présente une cohérence remarquable, malgré sa composition de textes éparpillés et fragmentaires; elle n'a pas pour autant survécu dans les courants philosophiques qui ont suivi. Nous ajouterons qu'elle s'inscrit naturellement dans le contexte néoplatonicien, où elle apporte à la fois une clarification des limites de notre approche cognitive du divin et un examen minitieux du fonctionnement de la partie rationnelle de cette activité.

Aikaterini Lefka (Université de Liège)

Giovanni Casadio, Vie gnostiche all'immortalità, Brescia, Editrice Morcelliana, 1997. 1 vol. $15,5 \times 22,5 \mathrm{~cm}, 107$ p. (Coll. Letteratura cristiana antica). ISBN : 88-373-1648-3.

Cet ouvrage comporte deux chapitres qui dérivent de deux publications antérieures et répond à une exigence de relier entre elles deux contributions présentées en des circonstances différentes. L'auteur entend les articuler par une approche phénoménologique le distinguant de ses prédécesseurs qui, cherchant les origines de la gnose, sont arrivés à des conclusions diverses. Le premier cha pitre repose le problème de la gnose séthienne. Après avoir rappelé les thèses de Pearson et d'Onasch qui avaient remis en question l'assimilation de Seth, fils d'Adam, et du dieu égyptien, l'auteur reprend les différents témoignages à propos des Séthiens afin de déterminer s'ils ont une réalité historique. La question de la reconnaissance de l'hérésie séthienne a en effet divisé les spécialistes qui se partagent en « anti-séthiens » déclarés (Tardieu, Rudolph et Wisse) et en théoriciens du gnosticisme séthien (Schenke et Turner). Les premiers ont insisté sur l'inorganisation et l'incohérence du système séthien tel qu'il est exposé dans trois chapitres du Refutatio omniun baeresium d'Hippolyte. L'auteur en donne une traduction et un commentaire dans lequel il met en évidence que l'ontologie séthienne, bien que présentant une conception tripartite de l'univers, est en réalité fondée sur un dualisme radical d'un type peu fréquent dans les systèmes gnostiques : lumière et ténèbres y sont conçus comme des principes opposés dès l'origine et inconciliables par essence. Ce dualisme que l'auteur, reprenant l'ancienne thèse de Bousset, rattache au modèle perse distingue la doctrine séthienne des Gnostiques d'Irénée et des Séthiens notés dans d'autres sources hérésiologiques. La reconnaissance d'une gnose séthienne viendrait encore de l'invitation par Hippolyte à recourir à un livre intitulé Parapbrases de Seth, dont l'auteur étudie les rapports avec les Paraphrases de Sem de Nag Hammadi. Enfin, Casadio étudie la possibilité d'une source orphique puisque, aux dires d'Hippolyte, toute la doctrine dérive des antiques théologiens, Musée et Orphée. Il décèle entre les Orphiques et les Séthiens une thématique proche fondée sur la distinction entre le monde de la lumière (mâle) et celui des ténèbres (matrice femelle), ainsi qu'une pratique rituelle analogue, mais n'en souligne pas moins l'écart de six siècles entre les deux mouvements. Toutefois, il ne considère pas non plus qu'Hippolyte aurait donné une interprétation orphique de la doctrine séthienne à seule fin de la flétrir en la rattachant à une origine païenne. Le titre du second chapitre, la via della trasgressione in ipo e in iper, presque sibyllin aux dires de l'auteur, suggère que les gnostiques chrétiens prônent une ascèse dont le but est 